\title{
Anti-Caries Effect of Arginine-Containing Formulations in vivo: A Systematic Review and Meta-Analysis
}

\author{
Jialing Li ${ }^{\mathrm{a}} \quad$ Ziwei Huang ${ }^{\mathrm{b}} \quad \mathrm{Li} \mathrm{Mei}^{\mathrm{c}} \quad$ Guifeng $\mathrm{Li}^{\mathrm{a}}$ Huang $\mathrm{Li}^{\mathrm{b}}$ \\ Departments of ${ }^{a}$ Orthodontics and ${ }^{b}$ Oral Sciences, Nanjing Stomatological Hospital, Medical School of \\ Nanjing University, Nanjing, China; ' $D$ iscipline of Orthodontics, Department of Oral Science, Faculty of Dentistry, \\ University of Otago, Dunedin, New Zealand
}

\section{Key Words}

Arginine $\cdot$ Caries · Systematic review

\begin{abstract}
Objective: To assess the anti-caries effect of arginine-containing formulations in vivo on caries lesions compared with fluorides or placebo. Methods: Randomized or quasi-randomized human clinical trials wherein arginine was delivered by any method were considered. The MEDLINE, Web of Science, EMBASE, Cochrane Library, and CBM databases were searched to identify relevant articles published up to December 2014. Grey literature was also searched. Two authors performed data extraction independently and in duplicate using data collection forms. Each included study was assessed using the Cochrane risk of bias assessment tool. $\boldsymbol{R e}$ sults: Of the 470 studies screened, 31 full articles were scrutinized and assessed for eligibility. Ten studies ( $n=15,546$ participants) were selected for final inclusion. The metaanalysis results ( $\mathrm{n}=7$ studies) demonstrated a synergistic effect of arginine when used in conjunction with fluoride on early coronal and root caries compared with placebo or fluoride alone. No specific side effects related to arginine usage were identified. Conclusions: When used in combination with a calcium compound and fluoride, arginine potentially
\end{abstract}

provides a superior anti-caries effect compared with matched formulations of fluoride alone. However, the level of evidence was downgraded because of risks of bias and potential publication bias. In the future, more high quality, nonindustry-supported clinical studies in this research area are required before any definitive recommendations can be made.

(c) 2015 S. Karger AG, Basel

As a global health concern, dental caries is a multifactorial disease and a dynamic process that can be arrested and reversed during the early stage [Shay, 1997; ten Cate, 1997; Zimmer, 2001]. Fluoride is generally accepted as a caries-preventive agent. In recent decades, the extensive use of fluoride products, such as dentifrice or mouth rinses, has contributed to the dramatic decline in the prevalence and severity of dental caries worldwide.

However, despite the highly successful use of fluoride, caries remains a ubiquitous public health problem. One major reason why fluoride cannot completely prevent caries is that it does little to influence oral biofilms, an important modifiable factor in dental caries, to reduce

J. Li and Z. Huang contributed equally to this work.

\section{KARGER 125\%}

(c) 2015 S. Karger AG, Base

$0008-6568 / 15 / 0496-0606 \$ 39.50 / 0$

E-Mail karger@karger.com

www.karger.com/cre 
cariogenic challenge. Thus, conventional fluoride toothpastes and mouth rinses exhibit limitations for high-risk individuals, especially those with high plaque levels and frequent sugar intake [National Institute of Health Consensus Development Panel, 2001; Bowen, 2002; Taubman and Nash, 2006]. Various new anti-caries agents have been introduced to enhance or supplement the remineralizing effect of fluorides.

Arginine is a semi-essential amino acid found in various human salivary proteins or peptides. It is metabolized by non-pathogenic bacteria, such as Streptococcus sanguinis, via the arginine deiminase system to produce energy, ammonia and carbon dioxide [Wijeyeweera and Kleinberg, 1989]. The production of ammonia increases the local $\mathrm{pH}$ and neutralizes the effects of acidification from sugar metabolism, thereby promoting a more alkaline condition that is unfavourable to cariogenic bacteria and thus reducing the cariogenicity of oral biofilms. A new dentifrice containing 1.5\% arginine was developed based on the mechanism of action of arginine and is commercially available (Colgate-Palmolive, New York, N.Y., USA).

Arginine was first identified by Kleinberg et al. [1979] as a critical factor that increases $\mathrm{pH}$ in 1979. In the following decades, a considerable amount of research was conducted to assess the anti-caries effect of arginine both in vitro and in vivo [Kleinberg et al., 1982; Kanapka and Kleinberg, 1983; Traudt and Kleinberg, 1996; Kleinberg, 1999]. Several studies reported the mechanisms of arginine's effect on oral bacterial metabolism and cariogenic acid formation [Kanapka and Kleinberg, 1983; Nascimento et al., 2009]. Subsequent clinical studies conducted on both adults and children since 2000 provide further evidence of the anticaries efficacy of arginine-containing formulations [Acevedo et al., 2005, 2008; Hu et al., 2013b; Kraivaphan et al., 2013; Souza et al., 2013; Srisilapanan et al., 2013; Yin et al., 2013a, b; Li et al., 2015; Petersen et al., 2015]. Human in situ studies as well as randomized controlled clinical trials revealed that the new arginine-containing compound provided significantly greater benefits in arresting and reversing caries lesions compared with conventional fluoride toothpaste alone [Cantore et al., 2013]. Although a few literature reviews have been published in this area [Liu et al., 2012; Cummins, 2013a, b; ten Cate and Cummins, 2013], no systematic review/meta-analysis based on individual studies has been published to date.

Therefore, the aim of this systematic review was to assess the anti-caries effect of arginine-containing formulations in vivo compared with placebo and/or fluoride alone.

Anti-Caries Effect of Arginine on Early

Caries in vivo

\section{Materials and Methods}

Types of Studies

Human randomized or quasi-randomized clinical trials were included. Human in situ randomized controlled trials that had not been studied previously were also considered.

\section{Participant Characteristics}

Participants included healthy humans with/without carious lesions. No age/race/gender limits were implemented for the participants. Studies wherein participants had other chronic oral diseases or systemic diseases (e.g., patients with advanced periodontal disease, patients with Sjögren's syndrome, and radiation patients) were excluded.

\section{Intervention Types}

Studies using arginine formulations in any modality and comparing their effects with those of placebo or fluoride alone were included. Moreover, no restrictions were implemented regarding the dose, frequency, duration or method of administration.

\section{Study Outcomes}

The primary outcome was coronal/root caries increments or changes in the proportion of participants developing new caries during the study duration. For enamel white spot lesions [Fejerskov and Kidd, 2003], which are considered the first stage of dental caries, the primary study outcome was the extent/severity of newly developed white spot lesions. The secondary outcome was the side effects of using arginine, including discomfort, oral hygiene deterioration, dental staining and oral malodour.

\section{Search Strategy}

Detailed search strategies based on a search strategy developed for MEDLINE were developed for each database and revised appropriately. The search strategies included a combination of controlled vocabulary and free text terms (online supplementary appendix 1; for all online supplementary material, see www.karger.com/doi/10.1159/000435894). The following databases were searched to identify all relevant articles written in English: MEDLINE (via PubMed), Web of Science, EMBASE, and CENTRAL (The Cochrane Library). To reduce language bias, the CBM (Chinese Biological Medical) database was also searched to find articles written in Chinese. The literature search was first performed in May 2014 and was later updated on December 2, 2014 (fig. 1). During both searches, the references of the selected articles and relevant reviews were assessed to locate additional studies.

Unpublished literature was also searched in the following databases: ClinicalTrial.gov, National Research Register, OpenGrey, the World Health Organization's International Clinical Trial Registry Platform, Database of Abstracts of Reviews of Effects, Health Technology Assessment (HTA), Turning Research into Practice (TRIP) database, National Technical Information Service (NTIS), International Clinical Trials Registry Platform Search Portal, International Federation of Pharmaceutical Manufacturers and Associations (IFPMA) Clinical Trials Portal, International Standard Randomised Controlled Trial Number Register, UK National Research Register (NRR), Eli Lilly and Company Clinical Trial Registry, OpenSIGLE, Pharmaceutical Industry Clinical Trials Database (ABPI/CMR), Current Con- 
Fig. 1. Systematic review flow diagram.

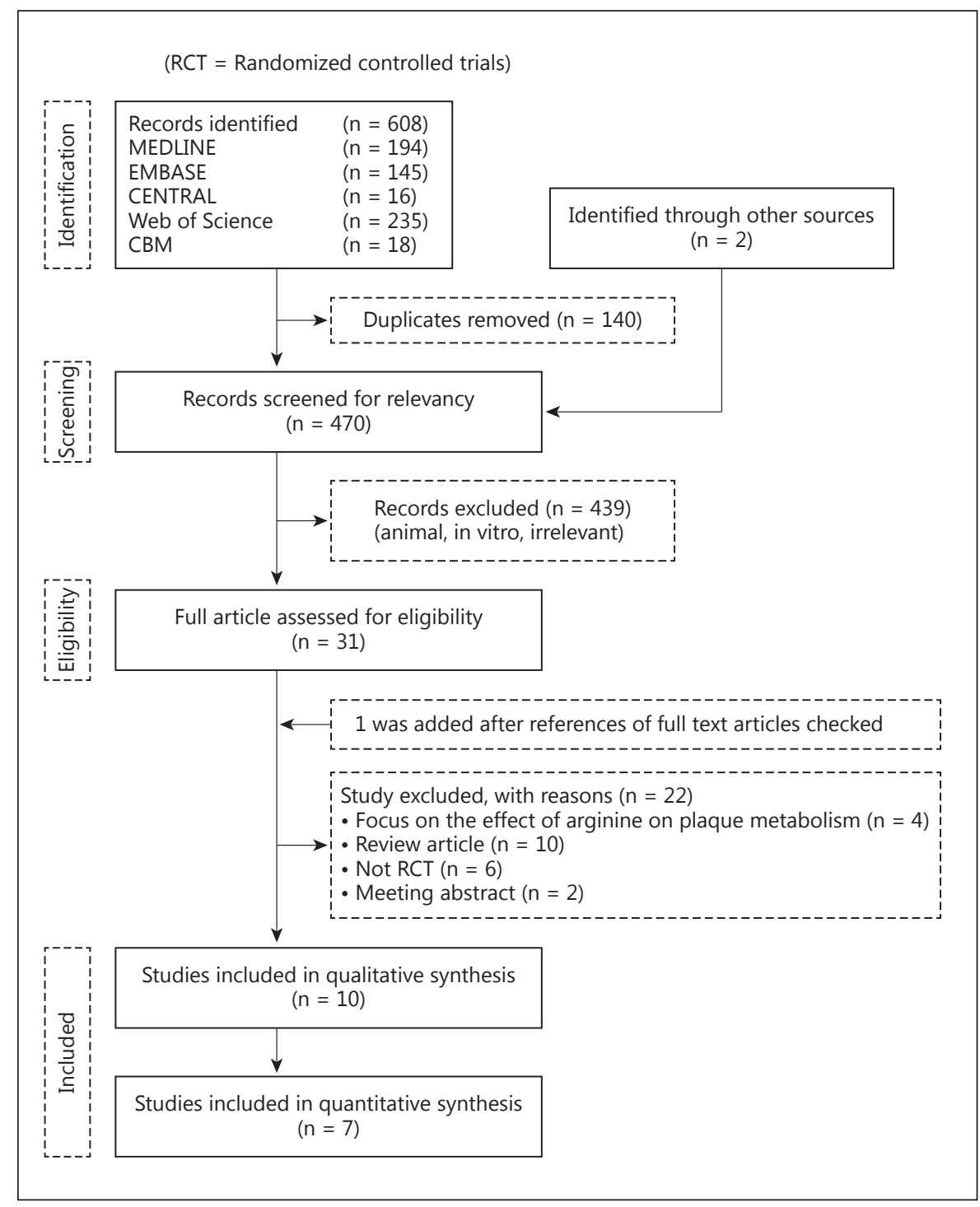
$\mathrm{RCT}=$ Randomized controlled trial.

trolled Trials and clinical study results for US-marketed pharmaceuticals. However, no related articles were identified in these databases.

\section{Data Collection and Analysis}

According to the predetermined inclusion and exclusion criteria, two calibrated reviewers independently screened the titles and abstracts (when available) of all identified studies in duplicate. Once a publication was considered by either author as meeting the inclusion criteria, the full-text article was obtained and scrutinized. If important information about a study was not available, the study authors were contacted by e-mail for additional information to resolve ambiguities, and their responses were accepted until May 20,2015 . Any disagreement during study selection and data extraction was resolved by discussion between the two reviewers; a third reviewer was consulted if necessary. Studies not meeting our inclusion criteria were excluded, and the reasons for their exclusion are noted in online supplementary appendix 2. Data extrac- tion was completed independently by the two reviewers using a specifically designed data extraction form. The following data were collected: authors and year of publication, number and age of participants, details of interventions and controls, follow-up period and assessment time points, assessment methods, and outcomes reported.

Each study was assessed using the evaluation method described in the Cochrane Handbook for Systematic Reviews of Interventions 5.1.0 (http://handbook.cochrane.org). The domains evaluated included random sequence generation, allocation concealment, blinding of participants and personnel, blinding of outcome assessment, incomplete outcome data, selective outcome reporting and other biases. The examiners assessed the presence of conflict of interest by reviewing the authors' disclosures and acknowledgements in the manuscript according to the criterion used by Friedman and Richter [2004]. A conflict of interest was defined as any financial relationship with companies that had their products evaluated in the study. 
Mean differences and standard deviations (SDs) were used to summarize data with continuous outcomes, whereas risk ratios and 95\% confidence intervals (CIs) were used for studies with dichotomous outcomes. Meta-analyses were performed only if sufficient similarities existed among the included studies regarding the types of participants, interventions, outcomes, and time points of the outcome measurements. The Grading of Recommendations, Assessment, Development and Evaluation (GRADE) system was used to assess the quality of the body of evidence in relation to review questions via GRADEprofiler 3.6 software [Guyatt et al., 2008].

\section{Results}

Of the 470 articles screened for relevancy, 31 full articles were scrutinized and assessed for eligibility. These 31 full articles included 2 recently published studies identified through other sources [Li et al., 2015; Petersen et al., 2015] (fig. 1). Finally, 10 studies (all in English) [Acevedo et al., 2008; Cantore et al., 2013; Hu et al., 2013b; Kraivaphan et al., 2013; Souza et al., 2013; Srisilapanan et al., 2013; Yin et al., 2013a, b; Li et al., 2015; Petersen et al., 2015] met the inclusion criteria and were used in the systematic review (table 1).

\section{Study Characteristics}

Of the 10 articles included in this review, 9 were randomized controlled clinical trials [Acevedo et al., 2008; Hu et al., 2013b; Kraivaphan et al., 2013; Souza et al., 2013; Srisilapanan et al., 2013; Yin et al., 2013a, b; Li et al., 2015; Petersen et al., 2015], and 1 was a randomized controlled in situ study [Cantore et al., 2013]. A total of 15,546 participants were evaluated, all of whom were classified as healthy by the authors of the studies. All 10 studies reported the age and number of participants. Most of the participants were children or adolescents (6-14 years) [Acevedo et al., 2008; Kraivaphan et al., 2013; Srisilapanan et al., 2013; Yin et al., 2013a, b; Li et al., 2015; Petersen et al., 2015]. In addition, 695 participants were middle-aged or elderly [Cantore et al., 2013; Hu et al., 2013b; Souza et al., 2013]. All studies were conducted on permanent teeth.

The study durations varied from 2 weeks to 24 months [Acevedo et al., 2008; Cantore et al., 2013; Hu et al., 2013b; Kraivaphan et al., 2013; Souza et al., 2013; Srisilapanan et al., 2013; Yin et al., 2013a, b; Li et al., 2015; Petersen et al., 2015], depending on the different outcome measures used to detect caries lesions. Studies using clinical examination (visual/tactile) as a diagnostic method typically employed longer observation periods ( $>1$ year) [Acevedo et al., 2008; Kraivaphan et al., 2013; Li et al., 2015; Peter-

Anti-Caries Effect of Arginine on Early

Caries in vivo sen et al., 2015] compared with those using digital instruments (e.g., microradiography/electrical caries monitor/ quantitative light-induced fluorescence, QLF) for diagnosis ( $\leq 6$ months) [Cantore et al., 2013; Hu et al., 2013b; Srisilapanan et al., 2013; Yin et al., 2013a, b].

Arginine was marketed under the trade name of 'CaviStat ${ }^{\circledR}$ ' in 1 study, but the arginine concentration was not specified [Acevedo et al., 2008]. According to the authors and the Colgate-Palmolive Company, the anti-caries dentifrice used in the remaining 9 studies contained $1.5 \% \mathrm{w} / \mathrm{w}$ arginine, an insoluble calcium compound and 1,450 ppm fluoride [Cantore et al., 2013; Hu et al., 2013b; Kraivaphan et al., 2013; Souza et al., 2013; Srisilapanan et al., 2013; Yin et al., 2013a, b; Li et al., 2015; Petersen et al., 2015].

\section{Primary Study Outcomes}

Among the 10 studies included (table 1), 1 evaluated the effect of arginine on artificially demineralized human enamel thin sections in vivo. In this in situ study, arginine-containing test products were significantly more effective than the positive control, which only contained fluoride. No significant difference in efficiency was observed between the two arginine-containing products [Cantore et al., 2013].

The remaining 9 trials assessed the effect of arginine on natural carious lesions [Acevedo et al., 2008; Hu et al., 2013b; Kraivaphan et al., 2013; Souza et al., 2013; Srisilapanan et al., 2013; Yin et al., 2013a, b; Li et al., 2015; Petersen et al., 2015]. All of the trials concluded that the dentifrices containing $1.5 \%$ arginine, an insoluble calcium compound and 1,450 ppm fluoride provided significantly greater protection against early-stage caries [Acevedo et al., 2008; Hu et al., 2013b; Souza et al., 2013; Srisilapanan et al., 2013; Yin et al., 2013a, b] or caries lesion cavitation [Acevedo et al., 2008; Kraivaphan et al., 2013; Li et al., 2015; Petersen et al., 2015] compared with dentifrices containing fluoride alone.

The primary outcome measures used in the 10 studies included clinical assessment using the DMFS/DMFT index [Acevedo et al., 2008; Kraivaphan et al., 2013; Li et al., 2015; Petersen et al., 2015] or electrical caries monitor [Hu et al., 2013b], the detection of mineral density change from microradiography [Cantore et al., 2013], lesion hardness measurements [Hu et al., 2013b; Souza et al., 2013] and readings from fluorescence-based devices (QLF) [Srisilapanan et al., 2013; Yin et al., 2013a, b].

\section{Secondary Outcome of Studies}

Three studies [Kraivaphan et al., 2013; Li et al., 2015; Petersen et al., 2015] reported no occurrence of side ef- 


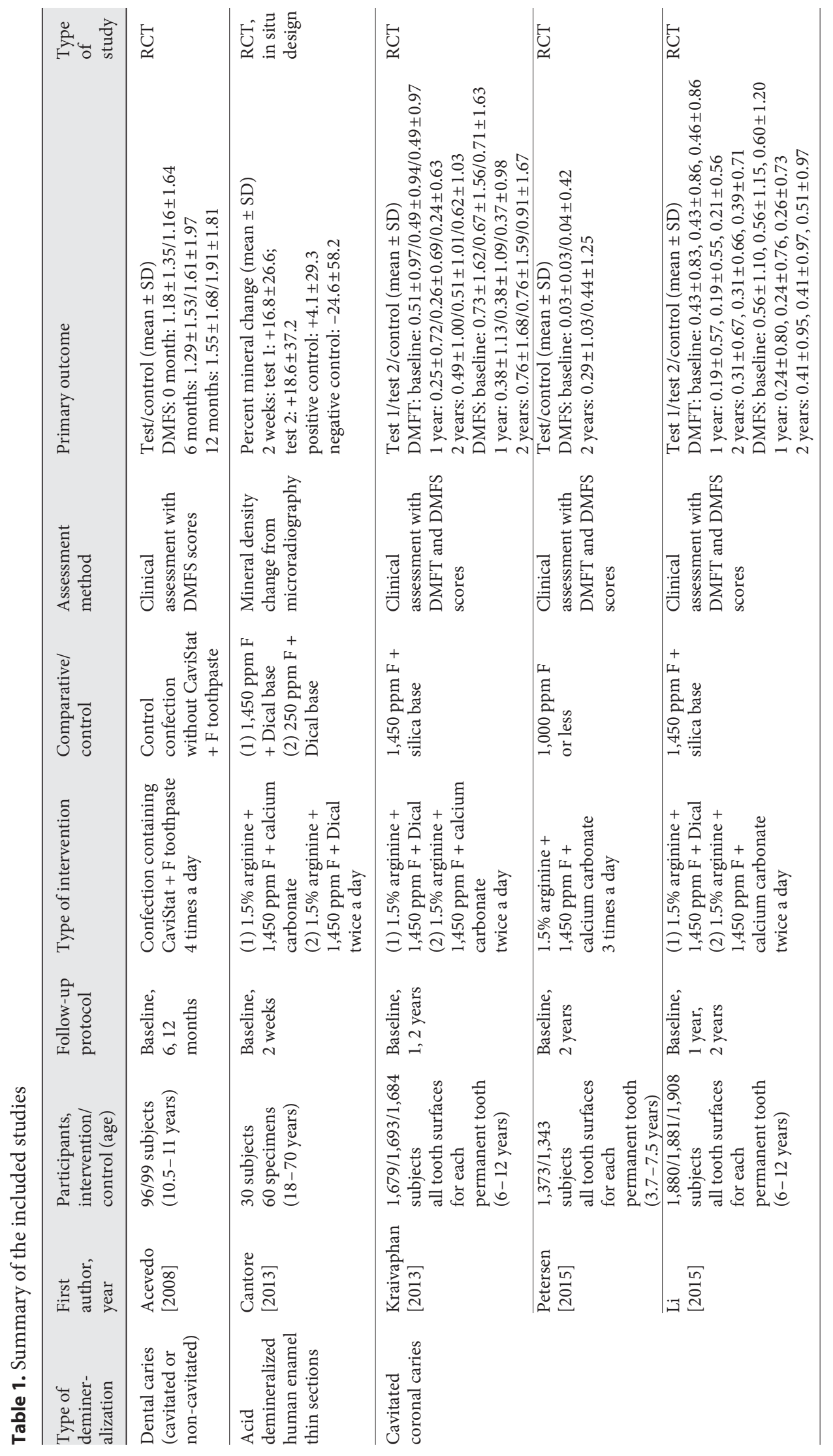




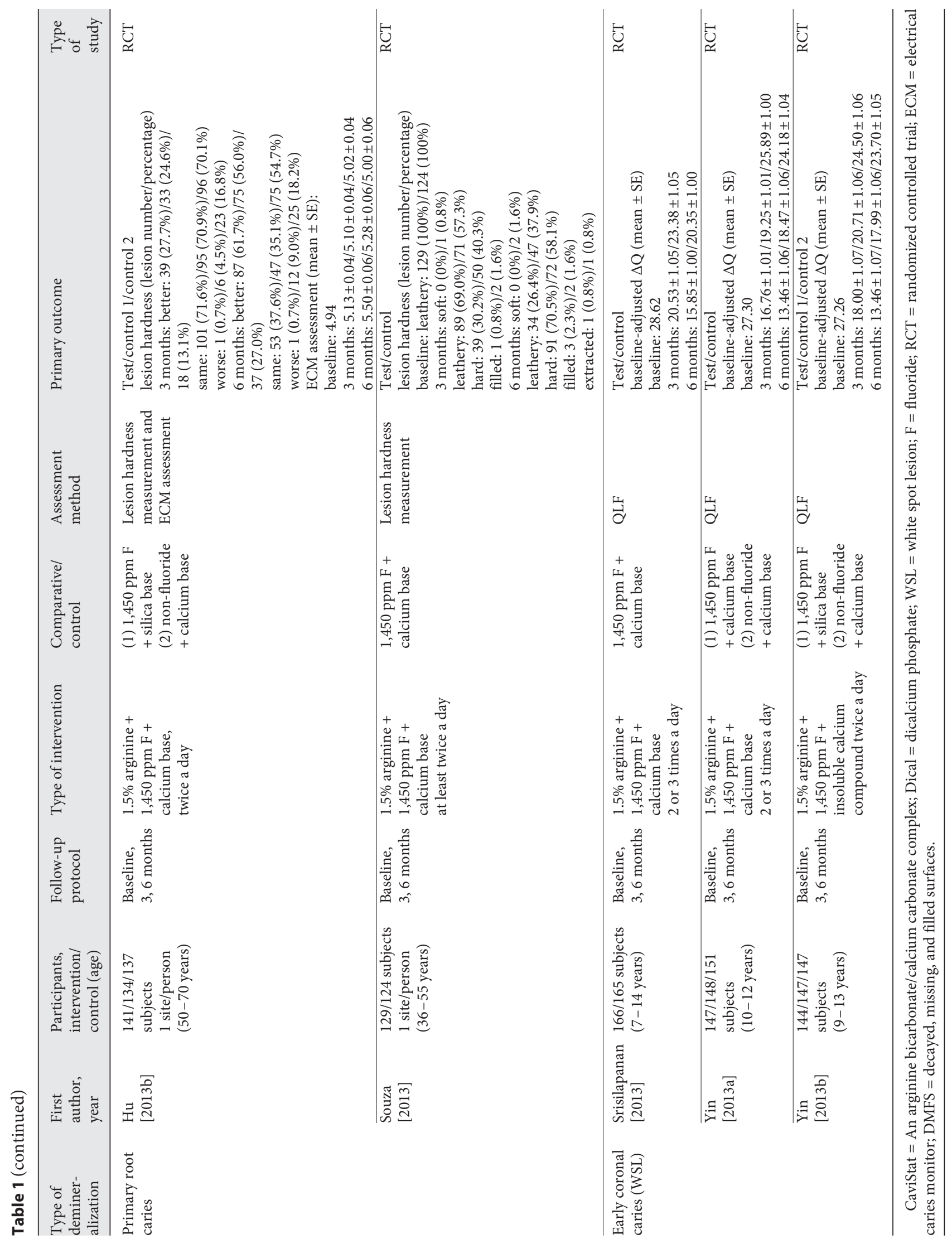


fects on oral hard/soft tissues in their 2-year follow-up trials. The authors of 2 studies also confirmed by e-mail correspondence that no significant differences were noted regarding the incidence of side effects (i.e., discomfort, headache, or allergies) between the intervention and control groups [Yin et al., 2013a, b]. The other 5 articles lacked information regarding the side effects of arginine [Acevedo et al., 2008; Cantore et al., 2013; Hu et al., 2013b; Souza et al., 2013; Srisilapanan et al., 2013].

\section{Risk of Bias}

Detailed risk of bias assessments for all included studies are summarized in figure 2 and in online supplementary appendix 3.

\section{Synthesis of Results}

Data were analysed with Review Manager 5.3. Forest plots for each meta-analysis were used to present the raw data (means, SDs and sample sizes) for each arm of the study. Studies rated as having high risk of bias [Acevedo et al., 2008; Petersen et al., 2015] were excluded from meta-analysis.

A dentifrice containing 1.5\% arginine, an insoluble calcium compound, and 1,450 ppm fluoride was the most common intervention [Cantore et al., 2013; Hu et al., 2013b; Kraivaphan et al., 2013; Souza et al., 2013; Srisilapanan et al., 2013; Yin et al., 2013a, b; Li et al., 2015; Petersen et al., 2015]. Three studies evaluated the efficacy of this dentifrice on early buccal coronal caries using QLF [Srisilapanan et al., 2013; Yin et al., 2013a, b], and 2 studies evaluated its efficacy on primary root caries [Hu et al., 2013b; Souza et al., 2013]. All of these 5 studies assessed the outcome at baseline as well as at 3 and 6 months of product use (table 1). Based on 270 and 258 carious lesions in the intervention and positive control groups, respectively, from the 2 studies related to the primary root caries, the summary risk ratio for $1.5 \%$ arginine was 1.16 (95\% CI: $1.01,1.33$ ) with low statistical heterogeneity (0.0\%) (fig. 3). Based on 457 and 460 carious lesions in the intervention and positive control groups, respectively, from the 3 studies related to early buccal coronal caries, the summary mean difference for $1.5 \%$ arginine was -4.67 (95\% CI: -6.34 , -3.01) (fig. 4). Two additional studies also used this compound as an intervention but were excluded from the meta-analysis due to different outcome measurements [Cantore et al., 2013] and a high risk of bias [Petersen et al., 2015]. The remaining 2 studies compared the effects of arginine dentifrices of different calcium bases with those of positive controls on cavitated coro-

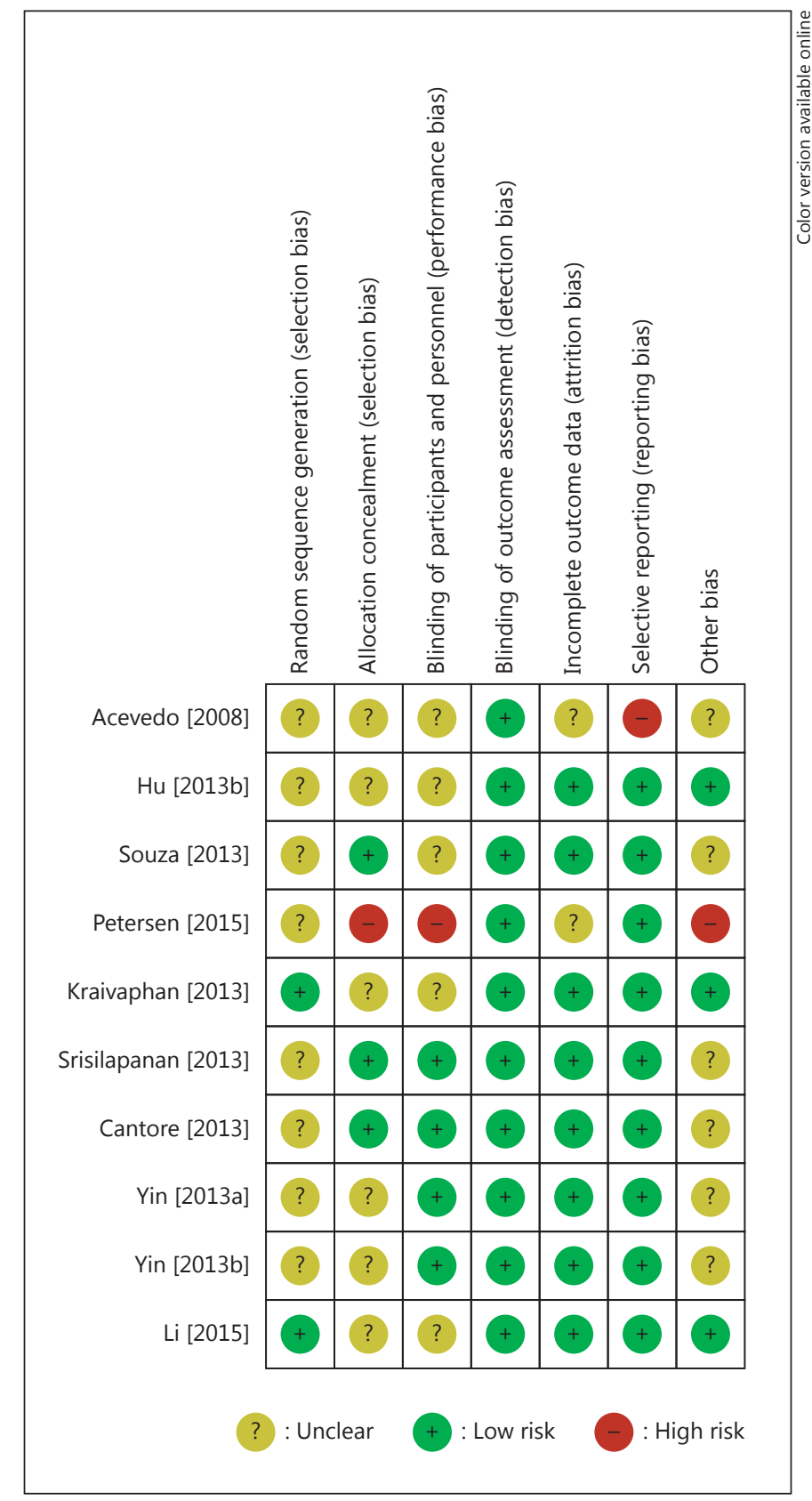

Fig. 2. Risk of bias assessment.

nal caries [Kraivaphan et al., 2013; Li et al., 2015]. Figures 5 and 6 show pooled mean differences of -0.09 (95\% CI: $-0.13,-0.06)$ and -0.09 (95\% CI: $-0.13,-0.05$ ), respectively. Another formulation was exclusively evaluated by a single study that concluded that CaviStat (an arginine bicarbonate/calcium carbonate complex) prevents the development of dental caries in teenagers [Acevedo et al., 2008]. 


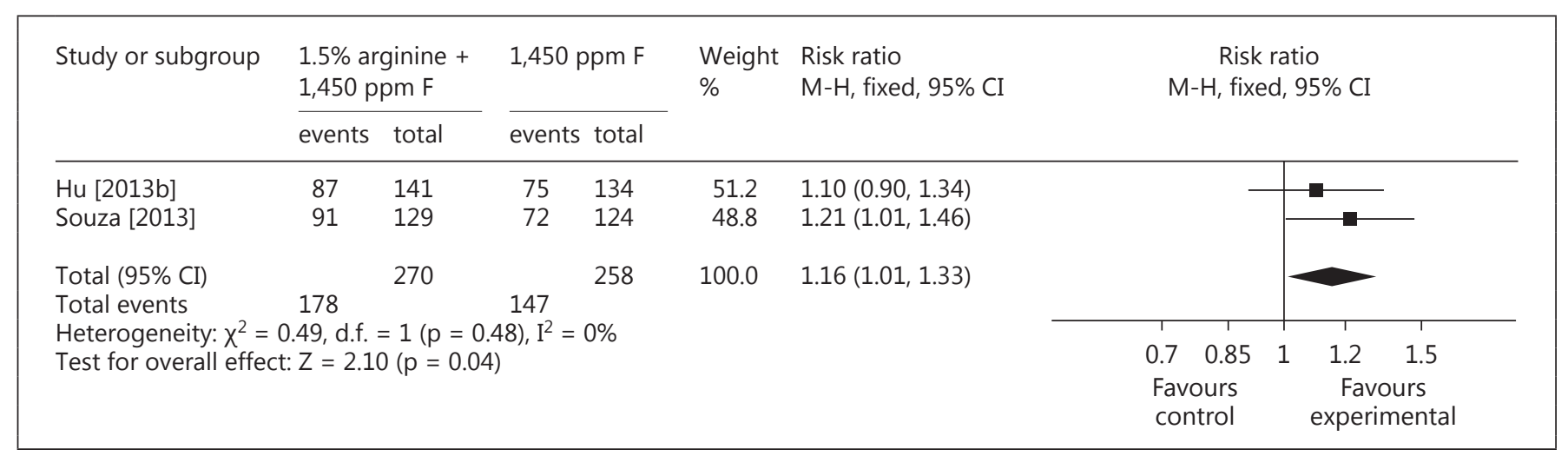

Fig. 3. Forest plot of comparison of $1.5 \%$ arginine $+1,450 \mathrm{ppm} F$ vs. $1,450 \mathrm{ppm}$ F for the event 'lesions improved after 6 months of follow-up'.

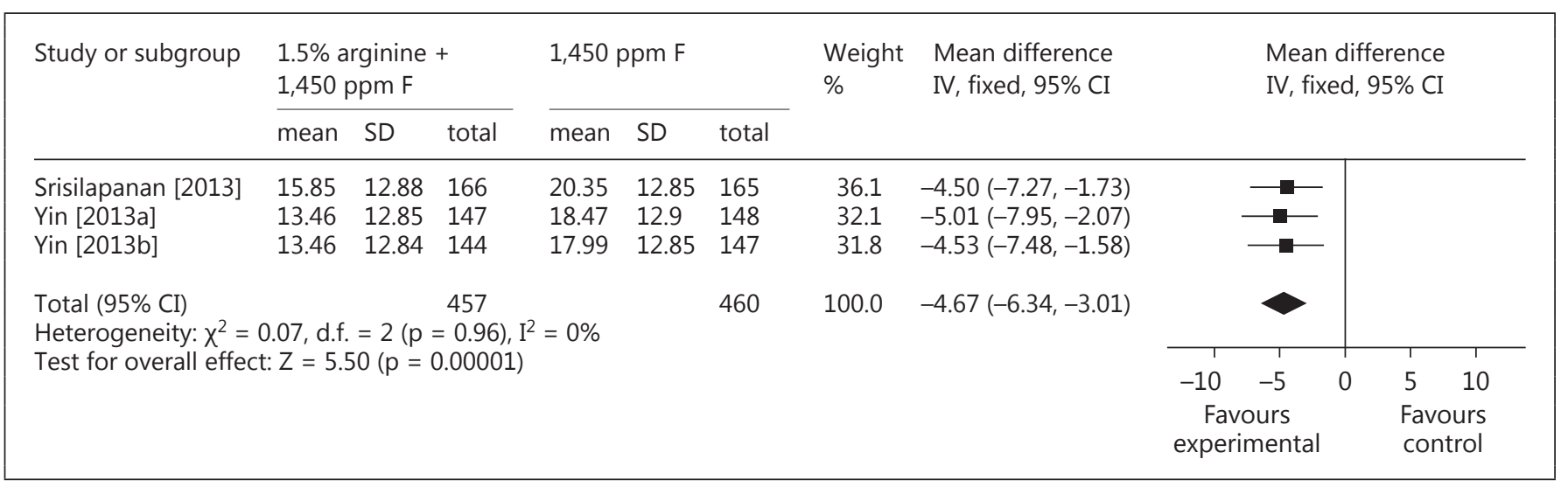

Fig. 4. Forest plot of comparison of $1.5 \%$ arginine $+1,450 \mathrm{ppm} F$ vs. $1,450 \mathrm{ppm} F$ for the mean subject $\Delta \mathrm{Q}$ after 6 months of follow-up.

\begin{tabular}{|c|c|c|c|c|c|c|c|c|c|c|}
\hline \multirow[t]{2}{*}{ Study or subgroup } & \multicolumn{3}{|c|}{$\begin{array}{l}1.5 \% \text { arginine }+ \\
1,450 \text { ppm } \mathrm{F}\end{array}$} & \multicolumn{3}{|c|}{1,450 ppm F } & \multirow[t]{2}{*}{$\begin{array}{l}\text { Weight } \\
\%\end{array}$} & \multirow[t]{2}{*}{$\begin{array}{l}\text { Mean difference } \\
\text { IV, fixed, } 95 \% \text { CI }\end{array}$} & \multicolumn{2}{|c|}{$\begin{array}{l}\text { Mean difference } \\
\text { IV, fixed, } 95 \% \text { CI }\end{array}$} \\
\hline & mean & $\mathrm{SD}$ & total & mean & SD & total & & & & \\
\hline Kraivaphan [2013] & 0.49 & 1 & 1,679 & 0.62 & 1.03 & 1,684 & 29.1 & $-0.13(-0.20,-0.06)$ & $\rightarrow$ & \\
\hline Li [2015] & 0.31 & 0.67 & 1,880 & 0.39 & 0.71 & 1,908 & 70.9 & $-0.08(-0.12,-0.04)$ & $\theta$ & \\
\hline \multirow{2}{*}{$\begin{array}{l}\text { Total }(95 \% \text { CI }) \\
\text { Heterogeneity: } x^{2}= \\
\text { Test for overall effe }\end{array}$} & 1.45 , d.f. & $=1(\mathrm{p}$ & $\begin{array}{l}3,559 \\
=0.23\end{array}$ & $=31 \%$ & & 3,592 & 100.0 & $-0.09(-0.13,-0.06)$ & $\diamond$ & \\
\hline & $6=3$. & $1(p-$ & .0 .0000 & & & & & & $\begin{array}{l}-0.5-0.250 \\
\text { Favours } \\
\text { experimental }\end{array}$ & $\begin{array}{c}0.25 \quad 0.5 \\
\text { Favours } \\
\text { control }\end{array}$ \\
\hline
\end{tabular}

Fig. 5. Forest plot of comparison of $1.5 \%$ arginine $+1,450 \mathrm{ppm}$ F/Dical vs. 1,450 ppm F for the DMFT score increments after 2 years of follow-up.

Anti-Caries Effect of Arginine on Early Caries in vivo 


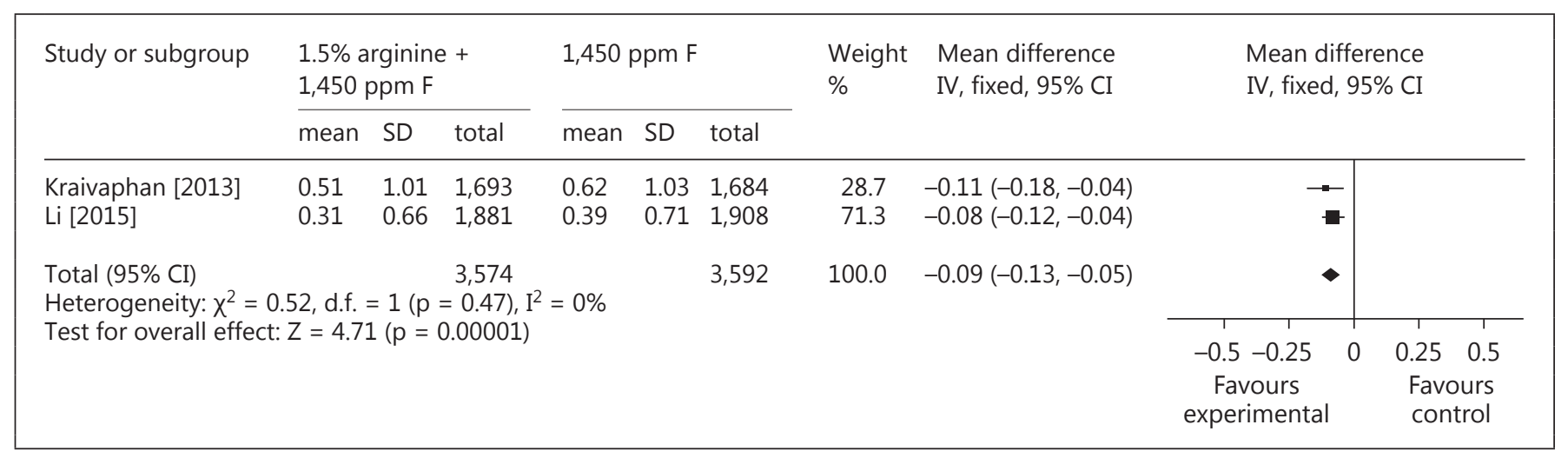

Fig. 6. Forest plot of comparison of $1.5 \%$ arginine $+1,450 \mathrm{ppm} F+$ calcium carbonate vs. $1,450 \mathrm{ppm} F$ for the DMFT score increments after 2 years of follow-up.

\section{Grading of Evidence}

Based on GRADE network levels, the level of the body of evidence was downgraded because of the risk of bias and potential publication bias (online suppl. appendix 4).

\section{Discussion}

A significant reduction in caries incidence was noted in the arginine-added intervention group compared with the control group; however, this result was based on a small number of clinical trials. Although both English and Chinese databases and the grey literature were systematically searched, only 10 studies were included in this systematic review.

One of the limitations of this systematic review is that all of the included studies were sponsored by product companies (1 was supported by Ortek Therapeutics [Acevedo et al., 2008], whereas the remaining 9 were sponsored by Colgate-Palmolive Company [Cantore et al., 2013; Hu et al., 2013b; Kraivaphan et al., 2013; Souza et al., 2013; Srisilapanan et al., 2013; Yin et al., 2013a, b; Li et al., 2015; Petersen et al., 2015]). In addition, some authors were also employees of the company [Cantore et al., 2013; Hu et al., 2013b; Kraivaphan et al., 2013; Souza et al., 2013; Srisilapanan et al., 2013; Yin et al., 2013a, b; Li et al., 2015; Petersen et al., 2015]. Industry sponsors may influence the outcome of a study in several ways. For example, sponsors may influence how the research question is framed, how the study is designed/conducted, and how the data are analysed; sponsors may also selectively report the results [Bero, 2013]. Greater than half of the protocols for industry-initiated trials stated that the sponsor either owned the data or needed to approve the manuscript or both [Gøtzsche et al., 2006]. In addition, industry-sponsored studies may have problems associated with multiple and selective publications [Melander et al., 2003], and these studies often lead to more favourable results and conclusions than the studies supported by other sources [Bekelman et al., 2003; Lexchin et al., 2003; Friedman and Richter, 2004]. The existence of such 'industry bias' in drug studies has also been confirmed by a recently published Cochrane systematic review [Lundh et al., 2012]. Therefore, when evaluating the quality of industry-funded studies, more reliable evaluations are likely obtained from assessing the raw data and methodological quality rather than completely relying on the authors' subjective interpretations.

In this systematic review, all 9 studies sponsored by the same company were similar in terms of study design, intervention/control arms, and time points [Cantore et al., 2013; Hu et al., 2013b; Kraivaphan et al., 2013; Souza et al., 2013; Srisilapanan et al., 2013; Yin et al., 2013a, b; Li et al., 2015; Petersen et al., 2015]; hence, similar assessments of risk of bias were identified. The 10 trials included in the review all reported significant results in favour of arginine; however, publication bias was highly suspected. Ideally, funnel plots should be generated to recognize the extent of publication bias, but the limited number of studies identified limits the feasibility of generating funnel plots in the review.

As a component of a new dentifrice, the concentration of arginine used in most clinical trials was $1.5 \% \mathrm{w} / \mathrm{w}$ [Cantore et al., 2013; Hu et al., 2013b; Kraivaphan et al., 2013; Souza et al., 2013; Srisilapanan et al., 2013; Yin et al., 2013a, b; Li et al., 2015; Petersen et al., 2015]. In 
1 study, arginine was added to a sugarless mint confection in the form of CaviStat (an arginine bicarbonate/calcium carbonate complex, Ortek Therapeutics, Roslyn, N.Y., USA) [Acevedo et al., 2008]. We e-mailed the corresponding author and company to inquire as to the specific concentration of arginine in CaviStat, but a response was not received after the review was completed.

Nine of the included studies used a dentifrice containing 1,450 or $1,000 \mathrm{ppm}$ fluoride alone in a calcium or silica base as a positive control [Cantore et al., 2013; Hu et al., 2013b; Kraivaphan et al., 2013; Souza et al., 2013; Srisilapanan et al., 2013; Yin et al., 2013a, b; Li et al., 2015; Petersen et al., 2015]. Among them, 4 studies also designed a dentifrice containing $250 \mathrm{ppm}$ or non-fluoride in a calcium base as a control [Cantore et al., 2013; Hu et al., 2013b; Yin et al., 2013a, b]. One study used a sugarless mint that contained all of the ingredients except arginine for the CaviStat as a negative control [Acevedo et al., 2008]. The use of non-fluoride or placebo control in caries trials may raise ethical issues, especially for patients with high caries risk. In consideration of patients' rights for oral health, non-fluoride or placebo control may only be used after very careful deliberation [Stamm, 2004]; for example, some studies have been conducted under strictly controlled conditions with regular examinations and supervised tooth brushing to ensure that no participant suffered negative effects [Yin et al., 2013a, b].

Many methods have been used to detect and monitor caries lesions. Traditional methods, such as clinical examination based on the DMFS index or ICDAS criteria and radiography, can only detect caries at the cavitation level and are not helpful at early caries stages. The objectivity and reproducibility of these traditional methods are poor [Pretty and Ellwood, 2013]. New approaches used in 4 of the included studies, such as QLF [Srisilapanan et al., 2013; Yin et al., 2013a, b] and the use of an electrical caries monitor [Hu et al., 2013b], can detect pre-cavitated lesions [Pretty, 2006; Alammari et al., 2013]. These new technologies enable the detection of early-stage caries and are both valid and highly reliable in quantifying the progression and reversal of carious lesions [Angmar-Mansson and ten Bosch, 1987; Hall et al., 1997; Shi et al., 2001; Baysan et al., 2004].

The follow-up of studies varied greatly, from 2 weeks to 2 years. Five of the included studies were conducted with a follow-up time of 6 months [Hu et al., 2013b; Souza et al., 2013; Srisilapanan et al., 2013; Yin et al., 2013a, b]. Three trials had a long follow-up time of 2 years [Kraivaphan et al., 2013; Li et al., 2015; Petersen et al., 2015]. With the development of new detection techniques

Anti-Caries Effect of Arginine on Early

Caries in vivo for early caries, the time needed to monitor caries formation has been reduced. However, some potential adverse effects of arginine usage still require a long follow-up [Hu et al., 2013a]. Given these factors, an observation period of no less than 6 months is typically suggested for human caries clinical trials (with the exception of in situ studies).

Arginine, a semi-essential amino acid found in many foods, such as milk and its by-products, is generally considered safe for use in toothpaste. Although no side effects were found in several clinical trials after 2 years or 6 months of follow-up, the potential association between the increased ammonia production from the breakdown of arginine and an increase in oral malodour remains poorly understood. Most studies have failed to present information regarding the potential side effects of arginine usage [Acevedo et al., 2008; Cantore et al., 2013; Hu et al., 2013b; Souza et al., 2013; Srisilapanan et al., 2013]. Safety assessments should be considered an important and necessary part of a well-designed randomized controlled trial [Higgins and Green, 2008].

One of the included studies did not consider the clustering effect [Cantore et al., 2013]. The assumption that tooth surfaces are independent within an individual can result in an underestimation of the standard error for treatment differences and an exaggerated $\mathrm{p}$ value for comparisons between the experimental and control groups [Burnside et al., 2006; Morgan et al., 2008]. The clustering effect should be considered either by calculating the sample size at the design stage of a trial or when analysing the data at the final stage of a trial [Campbell, 2004; Campbell et al., 2004].

In conclusion, within the limitation of this systematic review, evidence suggests that arginine in combination with calcium bases (either Dical or calcium carbonate) and fluoride provides a superior effect compared with fluoride alone. However, this conclusion was based on a small number of clinical trials, and potential publication bias could not be ignored. In the future, high-quality, welldesigned, non-industry-supported clinical studies are required before a definitive recommendation can be made.

\section{Acknowledgements}

This systematic review was supported by the National Natural Science Foundation of China (Nos. 81470712, 81070807). The authors thank Prof. R. Sullivan, Prof. R.P. Ellwood, Prof. W. Yin, Prof. D.Y. Hu and Prof. X. Li for providing copies of their articles or additional information about their studies. We gratefully acknowledge Prof. Jinhui Tian for his critical comments. We also express our gratitude to Prof. Liang Hong, who helped revise the manuscript. 


\section{Author Contributions}

Conceived and designed the study: J. Li, Z. Huang and H. Li. Literature search and data analysis: J. Li, Z. Huang and G. Li. Data interpretation and manuscript drafting: J. Li, Z. Huang, L. Mei and $\mathrm{H}$. Li. All authors critically revised the manuscript and gave final approval for the version to be published.

\section{Disclosure Statement}

This systematic review was supported by the National Natural Science Foundation of China (Nos. 81470712, 81070807). The funders had no role in the study design, data collection and analysis, decision to publish, or manuscript preparation. None of the authors have any conflicts of interest with respect to the authorship and/or publication of this article.

\section{References}

Acevedo AM, Machado C, Rivera LE, Wolff M, Kleinberg I: The inhibitory effect of an arginine bicarbonate/calcium carbonate CaviStat-containing dentifrice on the development of dental caries in Venezuelan school children. J Clin Dent 2005;16:63-70.

Acevedo AM, Montero M, Rojas-Sanchez F, Machado C, Rivera LE, Wolff M, Kleinberg I: Clinical evaluation of the ability of CaviStat in a mint confection to inhibit the development of dental caries in children. J Clin Dent 2008; 19:1-8.

Alammari MR, Smith PW, de Josselin de Jong E, Higham SM: Quantitative light-induced fluorescence (QLF): a tool for early occlusal dental caries detection and supporting decision making in vivo. J Dent 2013;41:127-132.

Angmar-Mansson B, ten Bosch JJ: Optical methods for the detection and quantification of caries. Adv Dent Res 1987;1:14-20.

Baysan A, Prinz JF, Lynch E: Clinical criteria used to detect primary root caries with electrical and mechanical measurements in vitro. Am J Dent 2004;17:94-98.

Bekelman JE, Li Y, Gross CP: Scope and impact of financial conflicts of interest in biomedical research: a systematic review. JAMA 2003;289: 454-465.

Bero L: Industry sponsorship and research outcome: a Cochrane review. JAMA Intern Med 2013;173:580-581.

Bowen WH: Do we need to be concerned about dental caries in the coming millennium? Crit Rev Oral Biol Med 2002;13:126-131.

Burnside G, Pine CM, Williamson PR: Statistical aspects of design and analysis of clinical trials for the prevention of caries. Caries Res 2006; 40:360-365

Campbell MJ: Extending consort to include cluster trials. BMJ 2004;328:654-655.

Campbell MK, Elbourne DR, Altman DG: Consort statement: extension to cluster randomised trials. BMJ 2004;328:702-708.

Cantore R, Petrou I, Lavender S, Santarpia P, Liu Z, Gittins E, Vandeven M, Cummins D, Sullivan R, Utgikar N: In situ clinical effects of new dentifrices containing $1.5 \%$ arginine and fluoride on enamel de- and remineralization and plaque metabolism. J Clin Dent 2013; 24(special No A):A32-A44.

Cummins D: The development and validation of a new technology, based upon $15 \%$ arginine, an insoluble calcium compound and fluoride, for everyday use in the prevention and treatment of dental caries. J Dent 2013a;41(suppl 2):S1-S11.

Cummins D: Dental caries: a disease which remains a public health concern in the 21 st century - the exploration of a breakthrough technology for caries prevention. J Clin Dent 2013b;24(special No A):A1-A14.

Fejerskov O, Kidd E: Dental Caries: The Disease and Its Clinical Management. Copenhagen, Blackwell Munksgaard, 2003.

Friedman LS, Richter ED: Relationship between conflicts of interest and research results. J Gen Intern Med 2004;19:51-56.

Gøtzsche PC, Hróbjartsson A, Johansen HK, Haahr MT, Altman DG, Chan AW: Constraints on publication rights in industry-initiated clinical trials. JAMA 2006;295:16451646.

Guyatt GH, Oxman AD, Vist GE, Kunz R, FalckYtter Y, Alonso-Coello P, Schunemann HJ: GRADE: an emerging consensus on rating quality of evidence and strength of recommendations. BMJ 2008;336:924-926.

Hall AF, DeSchepper E, Ando M, Stookey GK: In vitro studies of laser fluorescence for detection and quantification of mineral loss from dental caries. Adv Dent Res 1997;11:507-514.

Higgins JPT, Green S: Defining the review question and developing criteria for including studies; in O'Connor D, Green S, Higgins JPT (eds): Cochrane Handbook of Systematic Reviews, version 501. Oxford, Cochrane Collaboration, 2008, pp 83-94.

Hu D, Li X, Yin W, DeVizio W, Mateo LR, Dibart S, Zhang YP: Clinical investigation of oral malodor during long-term use of argininecontaining dentifrices. Am J Dent 2013a;26: 329-334.

Hu DY, Yin W, Li X, Feng Y, Zhang YP, Cummins D, Mateo LR, Ellwood RP: A clinical investigation of the efficacy of a dentifrice containing $1.5 \%$ arginine and $1,450 \mathrm{ppm}$ fluoride, as sodium monofluorophosphate in a calcium base, on primary root caries. J Clin Dent 2013b;24(special No A):A23-A31.

Kanapka J, Kleinberg I: Catabolism of arginine by the mixed bacteria in human salivary sediment under conditions of low and high glucose concentrations. Arch Oral Biol 1983;28: 1007-1015.

Kleinberg I: A new saliva-based anti-caries composition. Dent Today 1999;18:98-103.
Kleinberg I, Jenkins GN, Chatterjee R, Wijeyeweera L: The antimony $\mathrm{pH}$ electrode and its role in the assessment of dental plaque $\mathrm{pH}$. J Dent Res 1982;61:1139-1147.

Kleinberg I, Kanapka JA, Chatterjee R, Craw D, D'Angelo NK, Sandham HG: Metabolism of nitrogen by the oral mixed bacteria; in Kleinberg I, Ellison SA, Mandel ID (eds): Saliva and Dental Caries. Washington, Information Retrieval, 1979, pp 357-377.

Kraivaphan P, Amornchat C, Triratana T, Mateo LR, Ellwood R, Cummins D, DeVizio W, Zhang YP: Two-year caries clinical study of the efficacy of novel dentifrices containing $1.5 \%$ arginine, an insoluble calcium compound and 1,450 ppm fluoride. Caries Res 2013;47:582-590.

Lexchin J, Bero LA, Djulbegovic B, Clark O: Pharmaceutical industry sponsorship and research outcome and quality: systematic review. BMJ 2003;326:1167-1170.

Li X, Zhong YS, Jiang XJ, Hu DY, Mateo LR, Morrison $\mathrm{BM}$, Zhang YP: Randomized clinical trial of the efficacy of dentifrices containing $1.5 \%$ arginine, an insoluble calcium compound and 1,450 ppm fluoride over two years. J Clin Dent 2015;26:7-12.

Liu YL, Nascimento M, Burne RA: Progress toward understanding the contribution of alkali generation in dental biofilms to inhibition of dental caries. Int J Oral Sci 2012;4 135-140.

Lundh A, Sismondo S, Lexchin J, Busuioc OA, Bero L: Industry sponsorship and research outcome. Cochrane Database Syst Rev 2012; 12:MR000033.

Melander H, Ahlqvist-Rastad J, Meijer G, Beermann B: Evidence b(i)ased medicine - selective reporting from studies sponsored by pharmaceutical industry: review of studies in new drug applications. BMJ 2003;326:11711173

Morgan MV, Adams GG, Bailey DL, Tsao CE, Fischman SL, Reynolds EC: The anticariogenic effect of sugar-free gum containing CPPACP nanocomplexes on approximal caries determined using digital bitewing radiography. Caries Res 2008;42:171-184.

Nascimento MM, Gordan VV, Garvan CW, Browngardt CM, Burne RA: Correlations of oral bacterial arginine and urea catabolism with caries experience. Oral Microbiol Immunol 2009;24:89-95. 
National Institute of Health Consensus Development Panel: National Institutes of Health Consensus Development Conference statement. Diagnosis and management of dental caries throughout life, March 26-28, 2001. J Am Dent Assoc 2001;132:1153-1161.

Petersen PE, Hunsrisakhun J, Thearmontree A, Pithpornchaiyakul S, Hintao J, Jürgensen N, Ellwood RP: School-based intervention for improving the oral health of children in southern Thailand. Community Dent Health 2015;32:44-50.

Pretty IA: Caries detection and diagnosis: novel technologies. J Dent 2006;34:727-739.

Pretty IA, Ellwood RP: The caries continuum: opportunities to detect, treat and monitor the remineralization of early caries lesions. J Dent 2013;41(suppl 2):S12-S21.

Shay K: Root caries in the older patient: significance, prevention, and treatment. Dent Clin North Am 1997;41:763-793.

Shi XQ, Tranaeus S, Angmar-Mansson B: Comparison of QLF and DIAGNOdent for quantification of smooth surface caries. Caries Res 2001;35:21-26

Souza ML, Cury JA, Tenuta LM, Zhang YP, Mateo LR, Cummins D, Ellwood RP: Comparing the efficacy of a dentifrice containing $1.5 \%$ arginine and 1,450 ppm fluoride to a dentifrice containing $1,450 \mathrm{ppm}$ fluoride alone in the management of primary root caries. J Dent 2013;41(suppl 2):S35-S41.
Srisilapanan P, Korwanich N, Yin W, Chuensuwonkul C, Mateo LR, Zhang YP, Cummins D, Ellwood RP: Comparison of the efficacy of a dentifrice containing $1.5 \%$ arginine and 1,450 ppm fluoride to a dentifrice containing 1,450 ppm fluoride alone in the management of early coronal caries as assessed using quantitative light-induced fluorescence. J Dent 2013;41 (suppl 2):S29-S34.

Stamm JW: The classic caries clinical trial: constraints and opportunities. J Dent Res 2004; 83(special issue C):C6-C14.

Taubman MA, Nash DA: The scientific and public-health imperative for a vaccine against dental caries. Nat Rev Immunol 2006;6:555563.

ten Cate JM: Review on fluoride, with special emphasis on calcium fluoride mechanisms in caries prevention. Eur J Oral Sci 1997;105: 461-465.

ten Cate JM, Cummins D: Fluoride toothpaste containing $1.5 \%$ arginine and insoluble calcium as a new standard of care in caries prevention. J Clin Dent 2013;24:79-87.
Traudt M, Kleinberg I: Stoichiometry of oxygen consumption and sugar, organic acid and amino acid utilization in salivary sediment and pure cultures of oral bacteria. Arch Oral Biol 1996;41:965-978.

Wijeyeweera RL, Kleinberg I: Arginolytic and ureolytic activities of pure cultures of human oral bacteria and their effects on the $\mathrm{pH}$ response of salivary sediment and dental plaque in vitro. Arch Oral Biol 1989;34:43-53.

Yin W, Hu DY, Fan X, Feng Y, Zhang YP, Cummins D, Mateo LR, Pretty IA, Ellwood RP: A clinical investigation using quantitative lightinduced fluorescence (QLF) of the anticaries efficacy of a dentifrice containing $1.5 \%$ arginine and 1,450 ppm fluoride as sodium monofluorophosphate. J Clin Dent 2013a; 24(special No A):A15-A22.

Yin W, Hu DY, Li X, Fan X, Zhang YP, Pretty IA, Mateo LR, Cummins D, Ellwood RP: The anti-caries efficacy of a dentifrice containing $1.5 \%$ arginine and $1,450 \mathrm{ppm}$ fluoride as sodium monofluorophosphate assessed using quantitative light-induced fluorescence (QLF). J Dent 2013b;41(suppl 2):S22-S28.

Zimmer S: Caries-preventive effects of fluoride products when used in conjunction with fluoride dentifrice. Caries Res 2001;35(suppl 1): 18-21. 